\title{
C-MAP: Framework for Multi-agent Planning in Cyber Physical Systems
}

\author{
Sumant Mukherjee ${ }^{1}$ and Santanu Chaudhury ${ }^{2}$ \\ ${ }^{1}$ Institute for Systems Studies \& Analysis, DRDO, Delhi, India \\ \{sumantiitd, schaudhury\} @gmail.com \\ ${ }^{2}$ Dept. of Electrical Engineering, IIT, Delhi, India
}

\begin{abstract}
In this paper we propose a novel multi-agent planning framework that attempts to deal with dynamic nature of cyber physical system while planning and show usefulness of our approach in exploring different strategies and configurations of planning architecture. The proposed approach use multiagent planners at different layers of abstraction to collaborate by using concepts of task sharing, brokering of task allocation, continuous adaptive planning and blackboard based multi-agent interaction.
\end{abstract}

Keywords: cyber-physical system, multi-agent planning, task network, contract net protocol, blackboard.

\section{Introduction}

Cyber physical system (CPS) [1] intensively interact with physical processes where sensors sense the physical world, computational components process sensor data and activate actuators to influence the physical process in real time. The computational components for CPS need to be adaptive to deal with uncertainty of observations from sensor and outcome of actuator actions. Information-based warfare [2] is the term used in military circles to define network centric battle space that can be visualized as military CPS consisting of network of sensors, weapons and Command \& Control (C2) entities. Here the plans from multiple C2 planners need to be coordinated using various techniques of multi-agent planning (MAP) [3]. MAP for military CPS is an evolving technology for intelligent collaboration between multiple planner agents. Traditional decision-theoretic models become intractable when entities in CPS grow or the uncertainty about the environment increases.

This paper provides a novel MAP framework to deal with dynamic nature of CPS while planning using market-based algorithms [4], biologically inspired continuous planning [5] and blackboard-based [6] coordination between the planning layers. Domain of combat battlefield is used as case study to implement market-based algorithm for top-down task allocation to lower level planners. Bottom level planners use biologically inspired continuous planning to dynamically adapt to changing battlefield environment. Coordination between the planning layers is done using Blackboard. The effectiveness of the proposed approach has been illustrated using simulation testbed that generates typical military combat scenario. 
Section 2 provides an overview of proposed MAP framework and its components. Proposed approach along with application scope of market-based algorithm, continuous planning and blackboard-based coordination in MAP is described in section 3 . Section 4 shows the simulation experiments to illustrate the effectiveness of the proposed approach. Concluding remarks with future scope of the study are given in the last section.

\section{MAP for CPS: An Overview}

Planning problem in military CPS environment is a complex distributed problem solving task as it needs to harness inherently distributed resources and require communication and coordination between multiple C2 planners. We propose to deal with the complexity of distributed planning process in CPS environment using layers of abstraction for strategic, operational and tactical planning and collaboration between different layers of multi-agent planners. To construct a collective plan for working together, our higher level planning agents (strategic and operational planner) decompose task into sub-task (task decomposition), allocate sub-task to lower level planning agents (task allocation to operational and tactical planners), exchange sub-problem solution (result sharing) and synthesize solution using Blackboard (see Fig 1).

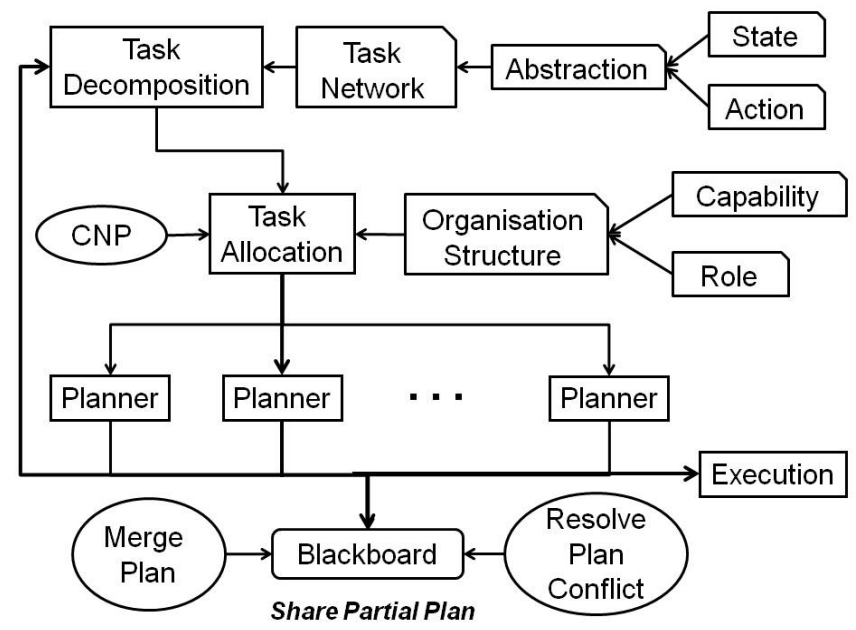

Fig. 1. Multi-Agent Planning Architecture

\section{Proposed Approach}

The paper proposes a unique MAP approach based on top-down concept of marketbased algorithms, bottom-up biologically inspired continuous planning and Blackboard-based coordination between the planning layers. Proposed Combat Multi-Agent Planner (C-MAP) implements layers of planners that reflect hierarchical organization 
of military (see Fig. 2). Global Commander (GC) is top level C2 layer that coordinates actions of the Local Commander (LC) which in turn coordinates actions of lowest level Unit commander (UC) under its command. C-MAP carries out major military tasks of surveillance, defensive and offensive operations in the simulated battlespace on need basis. For each operations GC generates set of operational tasks that all LC bids for and on allocation further decomposes the allocated operational task into tactical tasks. Task decomposition is carried out by making use of domain task network that consists of structured representation of set of tasks and inter-relationship between them based on state and action abstraction. The domain task network encode methods to achieving goals of surveillance, defense and offense by using operators to maneuver and engage threats, maintaining force balance, massing of units and helping peers in trouble.

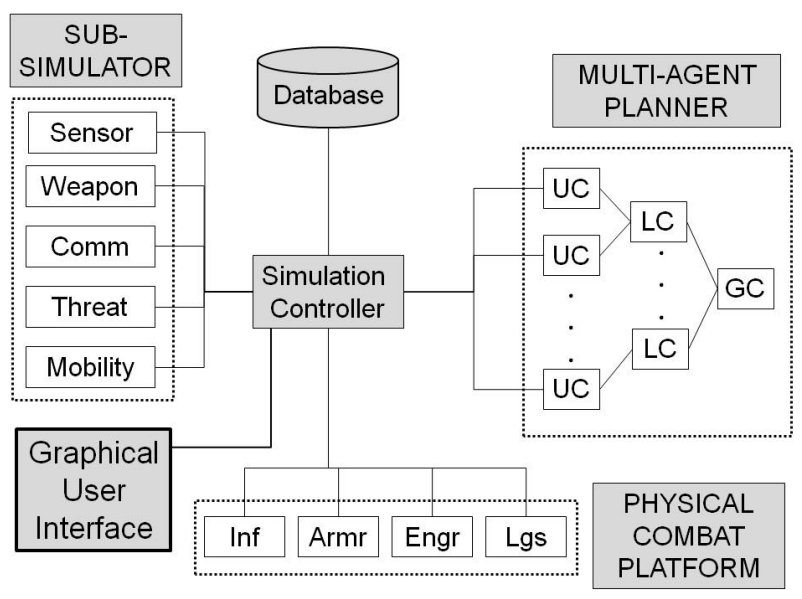

Fig. 2. Testbed Architecture

Market-based approach of contract net protocol (CNP) is used for task allocation in top-down fashion. Participants (LC or UC) bid for task depending on capability, role, current load of agents and task completion deadlines. Bidders use formally encoded multi-agent organizational structure that associates with each agent the types of tasks it can carry out and its priority over such tasks (this facilitates overlapping of agent capabilities). C-MAP multi-agent organizational structure encodes capabilities required to carry out a particular task, capabilities of particular type of agent and its prioritization over tasks

After successful task allocations, agents carry out adaptive continuous planning in bottom-up fashion using biologically inspired concept [5]. Combat is modeled as complex adaptive system where combat forces are visualized to be composed of large numbers of nonlinearly interacting parts. C-MAP agents continually adapt to changing combat environment and specifically adopt different "tendency" such as cluster with healthy friendly units, support injured friendly units, preserve self by avoiding healthy enemy units, be aggressive to injured enemy units and so on. 


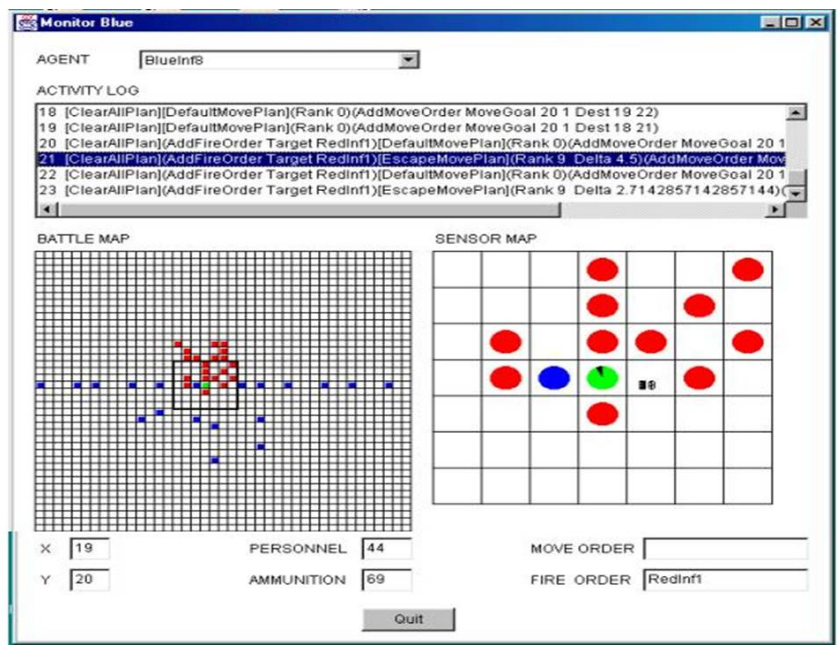

Fig. 3. C-MAP User Interface

Coordination between different layers of planners is achieved using Blackboard based inter-agent interaction. Different planner agents add structured knowledge tuple to Blackboard which others can query, update or delete. C-MAP Blackboard-Control agent directs the coordination process by managing how knowledge source (KS) agent responds to relevant event. KS agents act as critic of plans from different perspectives of task sharing opportunities, fusion of information, temporal consistency, and constraint violation. The task sharing critic agent looks for opportunities of sharing of common tasks. Possible opportunities could be achieving surveillance task as side-effect of offensive or defensive task, supplying of resources or clearing obstacles in proximity and so on. The task sharing critic agent identifies these opportunities by monitoring task related tuple such as agent active task assignment, task capabilities, agent capability and triggering task sharing event when matching rules fire. The fusion critic agent fuses lower level information to higher abstraction such as size of enemy-deployment (based on collocation of enemy units) or prediction of movevector of enemy-units (based on temporal tracking of enemy unit locations) [7]. The temporal critic agent monitor task with respect to its deadlines (gap between contracted task and actual state at end of contract) and trigger appropriate corrective actions such as additional operational or tactical tasks. The consistency critic agent checks for different constraint violation on tasks such as resource constraint such as unit does not have sufficient fuel or ammunition to carry out particular task. If a constraint violation is detected then consistency critic agent send "constraint-violation" message to relevant unit indicating that corrective action needs to be taken.

\section{Simulation Results}

We have implemented a combat simulation platform (CSP) to illustrate effectiveness of the proposed approach (see Fig. 2). CSP simulates typical military combat scenario 
in dynamic military CPS environment constituting of physical combat systems (PCS) with heterogeneous capability. Combat scenario gives details of total force composition of both blue and red side, their locations and resources such as sensors, weapons, communication devices, personnel, ammunition and fuel. Combat environment consists of terrain, weather and day/night characteristics where weather parameters effects sensor probabilities of detection $\mathrm{Pd}$, Weapon probability of kill $\mathrm{P}_{\mathrm{k}}$ and communication reliability. CSP provides PCS with limited local sensor information with uncertainty associated to it and restricted communication capability.

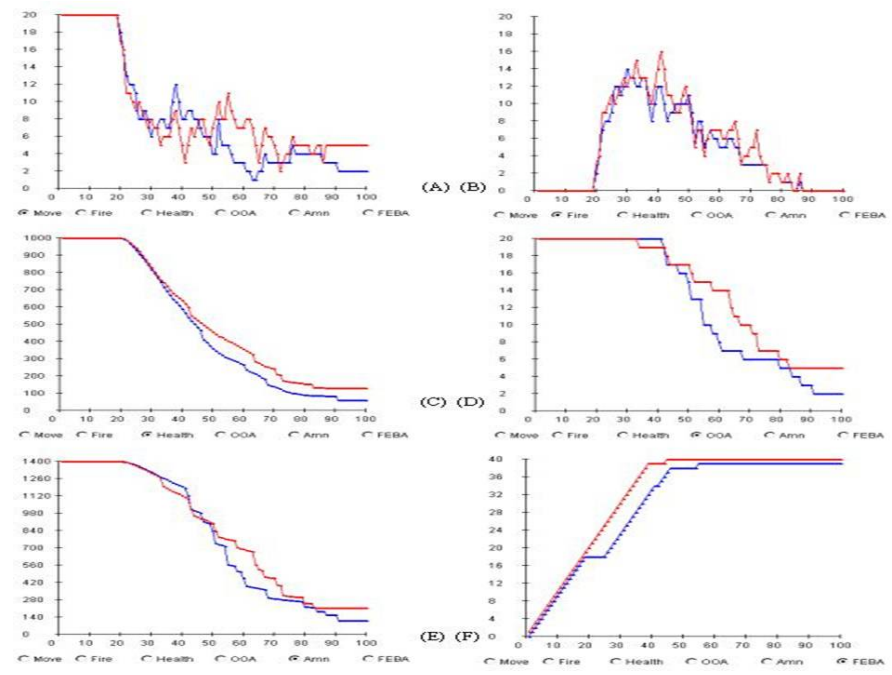

Fig. 4. Combat Action analysis curves

CSP constitutes of simulation controller (SC), sub-simulators and database module. C-MAP agents connect to respective PCS in CSP via SC. SC sends initial setting of the battlespace to each C-MAP agent. During simulation loop SC sends corresponding PCS sensor information to each agent and each agent can submit an action command to the SC individually. The SC sends agent action commands to sub-simulators (combat domain models for movement, threat, sensor, weapon, communication, obstacles, environment and attrition). These sub-simulators incorporate their respective effects and update the state of the battlespace. SC sends updated battlespace states to the GUI (see Fig. 3) and advances the simulation clock.

C-MAP can be configured to be completely decentralized (where units take independent decision without any C2 layer to guide them) or centralized C2 (i.e. units are guided by Global as well as local HQ). Fig. 4 depicts a simulation run output in term of combat actions taken by C-MAP agents of blue and red sides. The combat action analysis curves indicate effects of different configuration of C-MAP parameters on combat actions of maneuverability of units, casualties suffered, expenditure of resources etc. We believe that combination of CSP testbed and pluggable C-MAP multi-agent planning framework can be a useful tool for exploring different strategies for configurations for MAP architecture in CPS environment. 


\section{Conclusion}

In this paper we have proposed C-MAP, a novel multi-agent planning architecture to deal with dynamic nature of cyber-physical worlds where underlying planner adapts to dynamic initial state, goal state and planning domain. C-MAP use different abstraction layers of planning and use concepts of task network, CNP based negotiation, Blackboard-based agent interaction. The contribution of this paper lies in providing a framework to explore interplay between top-down marketplace dynamics and bottomup biologically inspired adaptive continuous planning to deal with dynamic CPS environment. To explore efficacy of C-MAP framework we have also implemented a testbed that simulates dynamic military CPS environment constituting of physical systems with heterogeneous capability. In conclusion it is worth observing that proposed framework is the first (to the best of our knowledge) that uses above ideas in a single framework. Our future work will focus on developing mechanism of semantic mediation to overcome heterogeneity challenges in CPS at different abstractions of physical devices, data/information and services using ontology model.

\section{References}

1. Tan, Y., Goddard, S.: A prototype architecture for cyber-physical systems. SIGBED Rev. 5(1), 1-2 (2008)

2. Alberts, D.S., et al.: Network Centric Warfare: Developing and Leveraging Information Superiority, 2nd edn. CCRP Press

3. Durfee, E.H.: Distributed Problem Solving and Planning. In: Weiss, G. (ed.), pp. 121-164. MIT Press, Cambridge (1999)

4. Smith, R.G.: The Contract Net Protocol. IEEE Trans. on Computers C-29(12) (December 1980)

5. Ilachinski, A.: Irreducible Semi-Autonomous Adaptive Combat (ISAAC): An Artificial-Life Approach to Land Warfare. Center for Naval Analyses Research Memorandum CRM 97-61 (June 1997)

6. Corkill, D.D.: Collaborating Software Blackboard and Multi-Agent Systems \& the Future. In: Proceedings of the International Lisp Conference (2003)

7. Gupta, M., Mukherjee, S.: Towards Situation Awareness in Integrated Air Defence Using Clustering and Case Based Reasoning. In: Chaudhury, S., Mitra, S., Murthy, C.A., Sastry, P.S., Pal, S.K. (eds.) PReMI 2009. LNCS, vol. 5909, pp. 579-584. Springer, Heidelberg (2009) 